\title{
Extremes of independent Gaussian processes
}

\section{Zakhar Kabluchko}

Received: 2 September 2009 / Revised: 17 February 2010 /

Accepted: 5 March 2010 / Published online: 6 April 2010

(C) Springer Science+Business Media, LLC 2010

\begin{abstract}
For every $n \in \mathbb{N}$, let $X_{1 n}, \ldots, X_{n n}$ be independent copies of a zeromean Gaussian process $X_{n}=\left\{X_{n}(t), t \in T\right\}$. We describe all processes which can be obtained as limits, as $n \rightarrow \infty$, of the process $a_{n}\left(M_{n}-b_{n}\right)$, where $M_{n}(t)=\max _{i=1, \ldots, n} X_{i n}(t)$, and $a_{n}, b_{n}$ are normalizing constants. We also provide an analogous characterization for the limits of the process $a_{n} L_{n}$, where $L_{n}(t)=$ $\min _{i=1, \ldots, n}\left|X_{i n}(t)\right|$.
\end{abstract}

Keywords Extremes - Gaussian processes - Max-stable processes ·

Hüsler-Reiss distributions

AMS 2000 Subject Classifications Primary-60G70; Secondary-60G15

\section{Introduction}

Suppose that we are given a large number $n$ of independent copies of some Gaussian process defined on an arbitrary set $T$. Let $M_{n}$ be the maximum of these processes, taken pointwise. The aim of this paper is to describe the class of processes which can be obtained as limits, as $n \rightarrow \infty$ and after suitable normalization, of the process

\footnotetext{
Z. Kabluchko ( $\square)$

Institut für Mathematische Stochastik, Georg-August-Universität Göttingen, Goldschmidtstraße 7, 37077 Göttingen, Germany

e-mail: kabluch@math.uni-goettingen.de,zakhar.kabluchko@uni-ulm.de
} 
$M_{n}$. To state our problem in a more precise way, let $X_{1 n}, \ldots, X_{n n}$ be $n$ independent copies of a zero-mean Gaussian process $\left\{X_{n}(t), t \in T\right\}$ with

$$
r_{n}\left(t_{1}, t_{2}\right):=\mathbb{E}\left[X_{n}\left(t_{1}\right) X_{n}\left(t_{2}\right)\right], \quad \sigma_{n}^{2}(t):=r_{n}(t, t)>0 .
$$

We define a process $\left\{M_{n}(t), t \in T\right\}$ by

$$
M_{n}(t)=\max _{i=1, \ldots, n} X_{i n}(t)
$$

Problem 1 Describe all sequences $X_{n}$ and all sequences of constants $a_{n}, b_{n}$ for which the process $a_{n}\left(M_{n}-b_{n}\right)$ converges as $n \rightarrow \infty$ to some nontrivial limit.

If not stated otherwise, convergence of stochastic processes is understood as the weak convergence of their finite-dimensional distributions.

Hüsler and Reiss (1989) (see also Example 4.1.4 in Falk et al. 1994) proved that $M_{n}$ converges as $n \rightarrow \infty$ to a nontrivial limiting process if the following two conditions are satisfied:

1. $\sigma_{n}(t)=1$ for all $t \in T$;

2. $\Gamma\left(t_{1}, t_{2}\right):=\lim _{n \rightarrow \infty} \log n \cdot\left(1-r_{n}\left(t_{1}, t_{2}\right)\right)$ exists in $(0, \infty)$ for all $t_{1}, t_{2} \in T$.

We will complement the work of Hüsler and Reiss (1989) by considering processes with non-unit variance, providing necessary and sufficient conditions for convergence, stressing the role of negative-definite kernels, and giving a different representation for the limiting processes. This representation is related to a symmetry property of certain exponential intensity Poisson point processes with Gaussian increments which is of independent interest. Also, we will provide a sufficient condition for the weak convergence of $M_{n}$ in the space of continuous functions.

Pointwise maxima of independent Gaussian processes have been studied by Brown and Resnick (1977) (who considered maxima of independent Brownian motions and Ornstein-Uhlenbeck processes) and by Kabluchko et al. (2009) (who considered stationary Gaussian processes on $\mathbb{R}^{d}$ ). We will extend some of the results of Kabluchko et al. (2009) to the non-stationary case.

We will also consider a similar problem for minima (always understood in the sense of absolute value) of independent Gaussian processes. With the above assumptions, we define a process $\left\{L_{n}(t), t \in T\right\}$ by

$$
L_{n}(t)=\min _{i=1, \ldots, n}\left|X_{i n}(t)\right|
$$

Problem 2 Describe all sequences $X_{n}$ and all sequences of constants $a_{n}, b_{n}$ for which the process $a_{n}\left(L_{n}-b_{n}\right)$ converges as $n \rightarrow \infty$ to some nontrivial limit.

The paper is organized as follows. In Section 2 we state and prove Theorem 1 which is needed to define the class of limiting processes for both maxima and minima 
of independent Gaussian processes. We solve Problem 1 and Problem 2 in Sections 3 and 4 , respectively. Sufficient conditions for tightness (in the space of continuous functions) of pointwise maxima of Gaussian processes are given in Section 5. As an illustration, we consider maxima and minima of independent fractional Brownian motions in Section 6.

\section{Negative-definite kernels and a family of Gauss-Poisson point processes}

\subsection{Negative-definite kernels}

We start by recalling some definitions related to negative-definite kernels; see Berg et al. (1984, Chapter 3). A kernel on a set $T$ is a mapping defined on $T \times T$. A kernel $\Gamma: T \times T \rightarrow[0, \infty)$ is called negative definite if for every $n \in \mathbb{N}, t_{1}, \ldots, t_{n} \in T$ and $a_{1}, \ldots, a_{n} \in \mathbb{R}$ satisfying $\sum_{i=1}^{n} a_{i}=0$, we have

$$
\sum_{i=1}^{n} \sum_{j=1}^{n} a_{i} a_{j} \Gamma\left(t_{i}, t_{j}\right) \leq 0 .
$$

It is well known that $d\left(t_{1}, t_{2}\right):=\sqrt{\Gamma\left(t_{1}, t_{2}\right)}$ defines a pseudo-metric on $T$. [This means that $d\left(t_{1}, t_{1}\right)=0, d\left(t_{1}, t_{2}\right)=d\left(t_{2}, t_{1}\right)$, and $d\left(t_{1}, t_{3}\right) \leq d\left(t_{1}, t_{2}\right)+d\left(t_{2}, t_{3}\right)$ for every $t_{1}, t_{2}, t_{3} \in T$, but, in general, $d\left(t_{1}, t_{2}\right)=0$ does not imply that $t_{1}=t_{2}$ ]. It is also known that a pseudometric $d$ on $T$ arises in this way for some negative-definite kernel $\Gamma$ if and only if the pseudometric space $(T, d)$ is isometrically embeddable into a Hilbert space.

Example 1 If $\{W(t), t \in T\}$ is a Gaussian process, then its incremental variance $\Gamma\left(t_{1}, t_{2}\right):=\operatorname{Var}\left(W\left(t_{1}\right)-W\left(t_{2}\right)\right)$ is a negative-definite kernel on $T$.

It will be convenient to use the following definition.

Definition 1 A kernel $\Gamma: T \times T \rightarrow[0, \infty]$ is called negative definite in the extended sense if there is a disjoint decomposition $T=\cup_{\alpha \in \mathcal{A}} T_{\alpha}$ such that the following two conditions are satisfied:

1. $\Gamma\left(t_{1}, t_{2}\right) \neq+\infty$ if and only if there is $\alpha \in \mathcal{A}$ such that $t_{1} \in T_{\alpha}$ and $t_{2} \in T_{\alpha}$;

2. For every $\alpha \in \mathcal{A}$, the restriction of $\Gamma$ to $T_{\alpha} \times T_{\alpha}$ is negative definite in the usual sense.

Note that the above conditions show that $\Gamma$ determines the decomposition $T=$ $\cup_{\alpha \in \mathcal{A}} T_{\alpha}$ uniquely. The next simple proposition establishes a link between positivedefinite and negative-definite kernels. 
Proposition 1 For each $n \in \mathbb{N}$, let $\left\{X_{n}(t), t \in T\right\}$ be a zero-mean, unit-variance Gaussian process with covariance function $r_{n}\left(t_{1}, t_{2}\right):=\mathbb{E}\left[X_{n}\left(t_{1}\right) X_{n}\left(t_{2}\right)\right]$. Suppose that there exists a sequence $z_{n}>0$ such that for all $t_{1}, t_{2} \in T$,

$$
\Gamma\left(t_{1}, t_{2}\right):=\lim _{n \rightarrow \infty} z_{n}\left(1-r_{n}\left(t_{1}, t_{2}\right)\right) \in[0, \infty]
$$

Then, the kernel $\Gamma$ is negative definite in the extended sense.

Proof Write $t_{1} \sim t_{2}$ if $\Gamma\left(t_{1}, t_{2}\right) \neq+\infty$. To see that " $\sim$ " is an equivalence relation on $T$, we need to show that if for some $t_{1}, t_{2}, t_{3} \in T$, both $\Gamma\left(t_{1}, t_{2}\right)$ and $\Gamma\left(t_{2}, t_{3}\right)$ are finite, then $\Gamma\left(t_{1}, t_{3}\right)$ is finite too. Let $d_{n}^{2}\left(t_{1}, t_{2}\right)=2 z_{n}\left(1-r_{n}\left(t_{1}, t_{2}\right)\right)$ be the incremental variance of the process $z_{n}^{1 / 2} X_{n}$. Note that $d_{n}^{2}$ is a negative-definite kernel and that $d_{n}$ is a pseudometric on $T$. If $\Gamma\left(t_{1}, t_{2}\right)$ and $\Gamma\left(t_{2}, t_{3}\right)$ are finite, then, by the triangle inequality,

$$
\sqrt{2 \Gamma\left(t_{1}, t_{3}\right)}=\lim _{n \rightarrow \infty} d_{n}\left(t_{1}, t_{3}\right) \leq \lim _{n \rightarrow \infty} d_{n}\left(t_{1}, t_{2}\right)+\lim _{n \rightarrow \infty} d_{n}\left(t_{2}, t_{3}\right)<\infty .
$$

Let $T=\cup_{\alpha \in \mathcal{A}} T_{\alpha}$ be the decomposition of $T$ into equivalence classes induced by the relation " $\sim$ ". To complete the proof, we need to show that for every $\alpha \in \mathcal{A}$, the restriction of $\Gamma$ to $T_{\alpha} \times T_{\alpha}$ is negative definite in the usual sense. This follows immediately from the fact that the limit of negative-definite kernels (provided it exists finitely) is negative definite.

\subsection{A family of Gauss-Poisson point processes}

In the next theorem we construct a family of exponential intensity Poisson processes related to each other by Gaussian increments. This construction will be used to define limiting processes for both maxima and minima of independent Gaussian processes. We refer to Resnick (1987, Chapter 3) for information on Poisson point processes.

Theorem 1 Fix $\lambda \in \mathbb{R}$ and a negative-definite kernel $\Gamma$ on a set $T$. Let $\left\{U_{i}, i \in \mathbb{N}\right\}$ be a Poisson point process on $\mathbb{R}$ with intensity $e^{-\lambda u} d u$, and let $W_{i}, i \in \mathbb{N}$, be independent copies of any zero-mean Gaussian process $\{W(t), t \in T\}$ with incremental variance $\Gamma$. Denote by $\sigma^{2}(t)$ the variance of $W(t)$. Define a function $V_{i}: T \rightarrow \mathbb{R}$ by

$$
V_{i}(t)=U_{i}+W_{i}(t)-\lambda \sigma^{2}(t) / 2 .
$$

Then, the law of the random family of functions $\left\{V_{i}, i \in \mathbb{N}\right\}$ depends only on $\Gamma$ and $\lambda$ (and does not depend on $\sigma^{2}$ ).

Remark 1 For a given negative-definite kernel $\Gamma$, there are many processes $W$ with incremental variance $\Gamma$. For example, for every $s \in T$ there is a unique in law 
zero-mean Gaussian process $W^{(s)}$ with incremental variance $\Gamma$ such that additionally, $W^{(s)}(s)=0$. The covariance function of this process is given by

$$
\mathbb{E}\left[W^{(s)}\left(t_{1}\right) W^{(s)}\left(t_{2}\right)\right]=\frac{1}{2}\left(\Gamma\left(t_{1}, s\right)+\Gamma\left(t_{2}, s\right)-\Gamma\left(t_{1}, t_{2}\right)\right) .
$$

Remark 2 We consider the random family of functions $\left\{V_{i}, i \in \mathbb{N}\right\}$ as a Poisson point process on the space $\mathbb{R}^{T}$ endowed with the product $\sigma$-algebra.

Remark 3 For every fixed $s \in T,\left\{V_{i}(s), i \in \mathbb{N}\right\}$ is a Poisson point process on $\mathbb{R}$ with intensity $e^{-\lambda u} d u$. [To see this, take $W=W^{(s)}$ in Theorem 1 . With this choice, we have $V_{i}(s)=U_{i}$ ]. This exponential intensity Poisson point process appears in the extreme-value theory as the limit for the extremal observations in an i.i.d. sample from the domain of attraction of the Gumbel distribution; see Section 4.4.2 in Resnick (1987). We may view the map $t \mapsto\left\{V_{i}(t), i \in \mathbb{N}\right\}$ as a family of dependent Poisson point processes on $\mathbb{R}$ with the same intensity related to each other via Gaussian jumps.

Remark 4 If $T=\mathbb{R}^{d}$ and $W$ is a process with stationary increments, the statement of Theorem 1 can be deduced from Kabluchko et al. (2009). In this case, the law of the family of functions $\left\{V_{i}, i \in \mathbb{N}\right\}$ is translation invariant.

\section{Proof of Theorem 1}

Step 1. Fix $s \in T$ and let $\left\{W^{(s)}(t), t \in T\right\}$ be a zero-mean Gaussian process defined as in Remark 1, i.e.

$$
W^{(s)}(s)=0, \quad \mathbb{E}\left[W^{(s)}\left(t_{1}\right) W^{(s)}\left(t_{2}\right)\right]=\frac{1}{2}\left(\Gamma\left(t_{1}, s\right)+\Gamma\left(t_{2}, s\right)-\Gamma\left(t_{1}, t_{2}\right)\right) .
$$

Let $\left\{V_{i}^{(s)}, i \in \mathbb{N}\right\}$ be the random collection of functions constructed as in Theorem 1 with $W=W^{(s)}$. More precisely, we define $V_{i}^{(s)}: T \rightarrow \mathbb{R}$ by

$$
V_{i}^{(s)}(t)=U_{i}+W_{i}^{(s)}(t)-\lambda \Gamma(t, s) / 2 .
$$

In Step 1 of the proof we are going to show that the law of the random family of functions $\left\{V_{i}^{(s)}, i \in \mathbb{N}\right\}$ does not depend on the choice of $s \in T$. To this end, we will modify an argument from Kabluchko et al. (2009).

Take some $t_{1}, \ldots, t_{k} \in T$. For a set $B \subset \mathbb{R}^{k}$ and $x \in \mathbb{R}$, denote by $B+x$ the diagonally shifted set $B+(x, x, \ldots, x)$. Let $\mathbb{P}_{t_{1}, \ldots, t_{k}}^{(s)}$ be the law of the random vector

$$
\left(W^{(s)}\left(t_{1}\right)-\lambda \Gamma\left(t_{1}, s\right) / 2, \ldots, W^{(s)}\left(t_{k}\right)-\lambda \Gamma\left(t_{k}, s\right) / 2\right),
$$


considered as a probability measure on $\mathbb{R}^{k}$. Then, we can view the random collection of points

$$
\left\{\left(V_{i}^{(s)}\left(t_{1}\right), \ldots, V_{i}^{(s)}\left(t_{k}\right)\right), i \in \mathbb{N}\right\}
$$

as a Poisson point process on $\mathbb{R}^{k}$ whose intensity $\Lambda_{t_{1}, \ldots, t_{k}}^{(s)}$ is given by

$$
\Lambda_{t_{1}, \ldots, t_{k}}^{(s)}(B)=\int_{\mathbb{R}} e^{\lambda x} \mathbb{P}_{t_{1}, \ldots, t_{k}}^{(s)}(B+x) d x, \quad B \in \mathcal{B}\left(\mathbb{R}^{k}\right) .
$$

Here, $\mathcal{B}\left(\mathbb{R}^{k}\right)$ is the $\sigma$-algebra of Borel subsets of $\mathbb{R}^{k}$. Consider a measure $\mu_{t_{1}, \ldots, t_{k}}^{(s)}$ on the $(k-1)$-dimensional hyperplane $\left\{\left(x_{i}\right)_{i=1}^{k} \in \mathbb{R}^{k}: x_{1}=0\right\}$ in $\mathbb{R}^{k}$, defined for $A \in \mathcal{B}\left(\mathbb{R}^{k}\right)$ by

$$
\mu_{t_{1}, \ldots, t_{k}}^{(s)}(A)=\int_{\mathbb{R}^{k}} e^{\lambda y_{1}} 1_{A}\left(0, y_{2}-y_{1}, \ldots, y_{k}-y_{1}\right) d \mathbb{P}_{t_{1}, \ldots, t_{k}}^{(s)}\left(y_{1}, \ldots, y_{k}\right) .
$$

Note that $\mu_{t_{1}, \ldots, t_{k}}^{(s)}\left(\mathbb{R}^{k}\right)=\mathbb{E} e^{\lambda W^{(s)}\left(t_{1}\right)-\lambda^{2} \Gamma\left(t_{1}, s\right) / 2}=1$, and therefore, $\mu_{t_{1}, \ldots, t_{k}}^{(s)}$ is a probability measure. As in Kabluchko et al. (2009), we can deduce from Eq. 11 that

$$
\Lambda_{t_{1}, \ldots, t_{k}}^{(s)}(B)=\int_{\mathbb{R}} e^{\lambda z} \mu_{t_{1}, \ldots, t_{k}}^{(s)}(B+z) d z, \quad B \in \mathcal{B}\left(\mathbb{R}^{k}\right) .
$$

Let $\psi_{t_{1}, \ldots, t_{k}}^{(s)}\left(u_{1}, \ldots, u_{k}\right)=\int_{\mathbb{R}^{k}} e^{\sum_{i=1}^{k} u_{i} y_{i}} d \mu_{t_{1}, \ldots, t_{k}}^{(s)}\left(y_{1}, \ldots, y_{k}\right)$ be the Laplace transform of the measure $\mu_{t_{1}, \ldots, t_{k}}^{(s)}$. It follows from Eq. 12 that

$$
\begin{aligned}
& \psi_{t_{1}, \ldots, t_{k}}^{(s)}\left(u_{1}, \ldots, u_{k}\right) \\
& =\int_{\mathbb{R}^{k}} e^{\lambda y_{1}} e^{u_{2}\left(y_{2}-y_{1}\right)+\ldots+u_{k}\left(y_{k}-y_{1}\right)} d \mathbb{P}_{t_{1}, \ldots, t_{k}}^{(s)}\left(y_{1}, \ldots, y_{k}\right) \\
& =\varphi_{t_{1}, \ldots, t_{k}}^{(s)}\left(\lambda-\sum_{i=2}^{k} u_{i}, u_{2}, \ldots, u_{k}\right)
\end{aligned}
$$

where $\varphi_{t_{1}, \ldots, t_{k}}^{(s)}=\int_{\mathbb{R}^{k}} e^{\sum_{i=1}^{k} u_{i} y_{i}} d \mathbb{P}_{t_{1}, \ldots, t_{k}}^{(s)}\left(y_{1}, \ldots, y_{k}\right)$ is the Laplace transform of the measure $\mathbb{P}_{t_{1}, \ldots, t_{k}}^{(s)}$. Recall that the measure $\mathbb{P}_{t_{1}, \ldots, t_{k}}^{(s)}$ is Gaussian with known covariance and expectation; see Eq. 10. Hence,

$$
\begin{aligned}
& \varphi_{t_{1}, \ldots, t_{k}}^{(s)}\left(u_{1}, \ldots, u_{k}\right) \\
& =\exp \left(-\frac{\lambda}{2} \sum_{i=1}^{k} \Gamma\left(t_{i}, s\right) u_{i}+\frac{1}{2} \sum_{i, j=1}^{k}\left(\Gamma\left(t_{i}, s\right)+\Gamma\left(t_{j}, s\right)-\Gamma\left(t_{i}, t_{j}\right)\right) u_{i} u_{j}\right) .
\end{aligned}
$$


An elementary calculation based on Eqs. 14 and 15 shows that

$$
\begin{aligned}
& \psi_{t_{1}, \ldots, t_{k}}^{(s)}\left(u_{1}, \ldots, u_{k}\right) \\
& =\exp \left(-\frac{\lambda}{2} \sum_{i=2}^{k} \Gamma\left(t_{i}, t_{1}\right) u_{i}+\frac{1}{2} \sum_{i, j=2}^{k}\left(\Gamma\left(t_{i}, t_{1}\right)+\Gamma\left(t_{j}, t_{1}\right)-\Gamma\left(t_{i}, t_{j}\right)\right) u_{i} u_{j}\right) .
\end{aligned}
$$

It follows that the measure $\mu_{t_{1}, \ldots, t_{k}}^{(s)}$ does not depend on $s \in T$. By Eq. 13, this implies that the measure $\Lambda_{t_{1}, \ldots, t_{k}}^{(s)}$ does not depend on $s$. Hence, the law of the random family of functions $\left\{V_{i}^{(s)}, i \in \mathbb{N}\right\}$ does not depend on the choice of $s \in T$.

Step 2. Now let $\{W(t), t \in T\}$ be a general zero-mean Gaussian process with incremental variance $\Gamma$ and variance $\sigma^{2}$. Our aim is to show that the law of the random family of functions $\left\{V_{i}, i \in \mathbb{N}\right\}$, where $V_{i}$ is defined as in Theorem 1, i.e.

$$
V_{i}(t)=U_{i}+W_{i}(t)-\lambda \sigma^{2}(t) / 2,
$$

does not depend on $\sigma^{2}$.

Let $\bar{T}$ be an extension of the set $T$ by a point $\tau_{0}$, i.e. $\bar{T}=T \cup\left\{\tau_{0}\right\}$. We extend the kernel $\Gamma$ to the set $\bar{T}$ as follows: let $\bar{\Gamma}\left(t_{1}, t_{2}\right)=\Gamma\left(t_{1}, t_{2}\right)$ if $t_{1}, t_{2} \in T ; \bar{\Gamma}\left(\tau_{0}, t\right)=$ $\bar{\Gamma}\left(t, \tau_{0}\right)=\sigma^{2}(t)$; and $\bar{\Gamma}\left(\tau_{0}, \tau_{0}\right)=0$. Then, $\bar{\Gamma}$ is a negative-definite kernel on $\bar{T}$. To see this, note that $\bar{\Gamma}$ is the incremental variance of the Gaussian process $\left\{\bar{W}^{\left(\tau_{0}\right)}(t), t \in\right.$ $\bar{T}\}$ defined by $\bar{W}^{\left(\tau_{0}\right)}\left(\tau_{0}\right)=0$, and $\bar{W}^{\left(\tau_{0}\right)}(t)=W(t)$ for $t \in T$.

Let $s \in \bar{T}$ be arbitrary. There is a unique zero-mean Gaussian process $\left\{\bar{W}^{(s)}(t), t \in \bar{T}\right\}$ with incremental variance $\bar{\Gamma}$ and $\bar{W}^{(s)}(s)=0$; see Remark 1. Step 1 of the proof implies that the law of the random family of functions $\left\{\bar{V}_{i}^{(s)}, i \in \mathbb{N}\right\}$, where $\bar{V}_{i}^{(s)}: \bar{T} \rightarrow \mathbb{R}$ is defined by

$$
\bar{V}_{i}^{(s)}(t)=U_{i}+\bar{W}_{i}^{(s)}(t)-\lambda \bar{\Gamma}(t, s) / 2,
$$

does not depend on $s \in T$ (here, $\bar{W}_{i}^{(s)}, i \in \mathbb{N}$, are independent copies of $\bar{W}^{(s)}$ ). Note that the restriction of the process $\bar{W}^{\left(\tau_{0}\right)}$ to $T$ has the same law as $W$, and that for $s \in T$, the restriction of $\bar{W}^{(s)}$ to $T$ has the same law as $W^{(s)}$. Thus, for $s \in T$, the restriction of the family $\left\{\bar{V}_{i}^{(s)}, i \in \mathbb{N}\right\}$ to $T$ has the same law as the family $\left\{V_{i}^{(s)}, i \in \mathbb{N}\right\}$ defined in Eq. 9, whereas the restriction of the family $\left\{\bar{V}_{i}^{\left(\tau_{0}\right)}, i \in \mathbb{N}\right\}$ to $T$ has the same law as the family $\left\{V_{i}, i \in \mathbb{N}\right\}$ defined in Eq. 16. It follows that the family $\left\{V_{i}, i \in \mathbb{N}\right\}$ has the same law as the family $\left\{V_{i}^{(s)}, i \in \mathbb{N}\right\}$, where $s \in T$ is arbitrary. Since the law of the latter family does not depend on $\sigma^{2}$, we obtain the statement of the theorem. 


\section{Maxima of independent Gaussian processes}

The main result of this section is Theorem 3 which solves Problem 1. Before we can state and prove it, we need to define the class of limiting processes in Section 3.1 and to recall a result of Hüsler and Reiss (1989) in a slightly generalized form and with a different representation of the limiting process in Section 3.2.

\subsection{Construction of limiting processes}

Let $\Gamma$ be a negative-definite kernel on a set $T$. Let $\left\{U_{i}, i \in \mathbb{N}\right\}$ be a Poisson point process on $\mathbb{R}$ with intensity $e^{-u} d u$, and let $W_{i}, i \in \mathbb{N}$, be independent copies of a zero-mean Gaussian process $\{W(t), t \in T\}$ with incremental variance $\Gamma$ and variance $\sigma^{2}$. Then, we define

$$
M_{\Gamma}(t)=\max _{i \in \mathbb{N}}\left(U_{i}+W_{i}(t)-\sigma^{2}(t) / 2\right) .
$$

Remark 5 By Theorem 1 with $\lambda=1$, the law of $M_{\Gamma}$ depends only on $\Gamma$.

Remark 6 A standard calculation with Poisson point processes shows that the finitedimensional distributions of $M_{\Gamma}$ are as follows: for every $t_{1}, \ldots, t_{k} \in T$ and $y_{1}, \ldots, y_{k} \in \mathbb{R}$, we have

$$
\begin{aligned}
\mathbb{P}\left[M_{\Gamma}\left(t_{1}\right) \leq\right. & \left.y_{1}, \ldots, M_{\Gamma}\left(t_{k}\right) \leq y_{k}\right] \\
& =\exp \left(-\int_{\mathbb{R}} \mathbb{P}\left[\exists j: u+W\left(t_{j}\right)-\sigma^{2}\left(t_{j}\right) / 2>y_{j}\right] e^{-u} d u\right) .
\end{aligned}
$$

An equivalent representation is given in Hüsler and Reiss (1989); see also Example 4.1.4 in Falk et al. (1994). Note that for every fixed $t \in T, M_{\Gamma}(t)$ is distributed according to the unit Gumbel distribution function $e^{-e^{-y}}$.

Remark 7 By construction, the process $M_{\Gamma}$ is max-stable; see de Haan (1984). More precisely, for every $k \in \mathbb{N}$, the process $\left\{\max _{i=1, \ldots, k} M_{\Gamma}^{(i)}(t), t \in T\right\}$, where $M_{\Gamma}^{(1)}, \ldots, M_{\Gamma}^{(k)}$ are independent copies of $M_{\Gamma}$, has the same law as $\left\{M_{\Gamma}(t)+\right.$ $\log k, t \in T\}$.

Example 2 Let $T=\mathbb{S}^{d}$ be the $d$-dimensional unit sphere and denote by $\rho$ the geodesic distance on $\mathbb{S}^{d}$. The kernel $\Gamma\left(t_{1}, t_{2}\right):=c \rho^{\beta}\left(t_{1}, t_{2}\right)$ is negative definite for $c>0, \beta \in(0,1)$; see Istas (2005). This leads to a family of rotationally invariant max-stable processes on $\mathbb{S}^{d}$ indexed by $c>0, \beta \in(0,1)$. A similar construction is possible on a space of constant negative curvature.

We need to slightly extend the definition of $M_{\Gamma}$ given above. Let $\Gamma$ be a negativedefinite kernel in the extended sense on a set $T$ with the corresponding decomposition 
$T=\cup_{\alpha \in \mathcal{A}} T_{\alpha}$. Then, we denote by $\left\{M_{\Gamma}(t), t \in T\right\}$ the stochastic process with the following properties:

1. For every $\alpha \in \mathcal{A}$, the process $\left\{M_{\Gamma}(t), t \in T_{\alpha}\right\}$ is defined as in Eq. 18;

2. For $\alpha, \beta \in \mathcal{A}$ with $\alpha \neq \beta$, the processes $\left\{M_{\Gamma}(t), t \in T_{\alpha}\right\}$ and $\left\{M_{\Gamma}(t), t \in T_{\beta}\right\}$ are independent.

\subsection{Sufficient conditions for convergence}

The next theorem is a slight modification of a result of Hüsler and Reiss (1989). Closely related results were obtained by Brown and Resnick (1977), Kabluchko et al. (2009). Let $u_{n}$ be a sequence such that

$$
\sqrt{2 \pi} u_{n} e^{u_{n}^{2} / 2} \sim n, \quad n \rightarrow \infty
$$

Equivalently, one can take

$$
u_{n}=\sqrt{2 \log n}-\frac{(1 / 2) \log \log n+\log (2 \sqrt{\pi})+o(1)}{\sqrt{2 \log n}}, \quad n \rightarrow \infty
$$

Theorem 2 For every $n \in \mathbb{N}$, let $X_{1 n}, \ldots, X_{n n}$ be independent copies of a zeromean, unit-variance Gaussian process $\left\{X_{n}(t), t \in T\right\}$ with covariance function $r_{n}\left(t_{1}, t_{2}\right):=\mathbb{E}\left[X_{n}\left(t_{1}\right) X_{n}\left(t_{2}\right)\right]$ such that for every $t_{1}, t_{2} \in T$,

$$
\Gamma\left(t_{1}, t_{2}\right):=\lim _{n \rightarrow \infty} 4 \log n \cdot\left(1-r_{n}\left(t_{1}, t_{2}\right)\right) \in[0, \infty] .
$$

Let $M_{n}(t)=\max _{i=1, \ldots, n} X_{i n}(t)$. Then, the process $u_{n}\left(M_{n}-u_{n}\right)$ converges as $n \rightarrow$ $\infty$ to the process $M_{\Gamma}$ in the sense of finite-dimensional distributions.

Remark 8 By Proposition 1, the kernel $\Gamma$ is negative definite in the extended sense. Hence, the process $M_{\Gamma}$ is well defined.

Remark 9 Hüsler and Reiss (1989) proved Theorem 2 assuming that $T$ is finite and that $\Gamma\left(t_{1}, t_{2}\right)<\infty$ for all $t_{1}, t_{2} \in T$, but they gave a different characterization of the limiting process.

Proof of Theorem 2 Suppose first that $\Gamma\left(t_{1}, t_{2}\right)<\infty$ for all $t_{1}, t_{2} \in T$. We will use an approach similar to that of Hüsler and Reiss (1989). This method is a first step of the "double sum method"; see Pickands (1969), Lemma 12.2.2 in Leadbetter et al. (1983), and Section D of Piterbarg (1996). Let $s \in T$ be arbitrary. It will be convenient to set $u_{n}(y)=u_{n}+u_{n}^{-1} y$ for $y \in \mathbb{R}$. Conditioned on the event $A_{n}(y):=\left\{X_{n}(s)=u_{n}(y)\right\}$, we have

$\mathbb{E}\left[u_{n}\left(X_{n}(t)-u_{n}\right) \mid A_{n}(y)\right]=u_{n}\left(u_{n}(y) r_{n}(t, s)-u_{n}\right)=y r_{n}(t, s)+u_{n}^{2}\left(r_{n}(t, s)-1\right)$. 
Taking into account Eqs. 21 and 22, we obtain

$$
\lim _{n \rightarrow \infty} \mathbb{E}\left[u_{n}\left(X_{n}(t)-u_{n}\right) \mid A_{n}(y)\right]=y-\frac{1}{2} \Gamma(t, s) .
$$

Further, by the well-known formula for the conditional covariance,

$$
\operatorname{Cov}\left[u_{n}\left(X_{n}\left(t_{1}\right)-u_{n}\right), u_{n}\left(X_{n}\left(t_{2}\right)-u_{n}\right) \mid A_{n}(y)\right]=u_{n}^{2}\left(r_{n}\left(t_{1}, t_{2}\right)-r_{n}\left(t_{1}, s\right) r_{n}\left(t_{2}, s\right)\right) .
$$

Together with Eqs. 21 and 22 this implies that

$$
\begin{aligned}
& \lim _{n \rightarrow \infty} \operatorname{Cov}\left[u_{n}\left(X_{n}\left(t_{1}\right)-u_{n}\right), u_{n}\left(X_{n}\left(t_{2}\right)-u_{n}\right) \mid A_{n}(y)\right] \\
& \quad=\frac{1}{2}\left(\Gamma\left(t_{1}, s\right)+\Gamma\left(t_{2}, s\right)-\Gamma\left(t_{1}, t_{2}\right)\right) .
\end{aligned}
$$

It follows from Eqs. 24 and 26 that for every fixed $y \in \mathbb{R}$, we have the following convergence of conditional processes

$$
\left\{u_{n}\left(X_{n}(t)-u_{n}\right) \mid A_{n}(y), t \in T\right\} \underset{n \rightarrow \infty}{\stackrel{f . d .}{\longrightarrow}}\left\{y+W^{(s)}(t)-\frac{1}{2} \Gamma(t, s), t \in T\right\} .
$$

We are going to compute the finite-dimensional distributions of $u_{n}\left(M_{n}-u_{n}\right)$ as $n \rightarrow \infty$. Fix some $k \in \mathbb{N}, t_{1}, \ldots, t_{k} \in T$, and $y_{1}, \ldots, y_{k} \in \mathbb{R}$. The density $f_{n}$ of the random variable $u_{n}\left(X_{n}(s)-u_{n}\right)$ is given by

$$
f_{n}(x)=\frac{1}{\sqrt{2 \pi} u_{n} e^{u_{n}^{2} / 2}} e^{-x} e^{-\frac{x^{2}}{2 u_{n}^{2}}}
$$

Conditioning on the event $A_{n}(y)$, we obtain

$$
\begin{aligned}
& \mathbb{P}\left[\exists j: X_{n}\left(t_{j}\right)>u_{n}\left(y_{j}\right)\right] \\
& \quad=\frac{1}{\sqrt{2 \pi} u_{n} e^{u_{n}^{2} / 2}} \int_{\mathbb{R}} \mathbb{P}\left[\exists j: u_{n}\left(X_{n}\left(t_{j}\right)-u_{n}\right)>y_{j} \mid A_{n}(y)\right] e^{-y} e^{-\frac{y^{2}}{2 u_{n}^{2}}} d y .
\end{aligned}
$$

It follows from Eqs. 20, 27, and a standard argument justifying the use of the dominated convergence theorem that

$$
\lim _{n \rightarrow \infty} n \mathbb{P}\left[\exists j: X_{n}\left(t_{j}\right)>u_{n}\left(y_{j}\right)\right]=\int_{\mathbb{R}} \mathbb{P}\left[\exists j: y+W^{(s)}\left(t_{j}\right)-\frac{1}{2} \Gamma\left(t_{j}, s\right)>y_{j}\right] e^{-y} d y .
$$

By the definition of $M_{n}$, we have

$$
\mathbb{P}\left[\forall j: u_{n}\left(M_{n}\left(t_{j}\right)-u_{n}\right) \leq y_{j}\right]=\left(1-\mathbb{P}\left[\exists j: X_{n}\left(t_{j}\right)>u_{n}\left(y_{j}\right)\right]\right)^{n} .
$$


It follows from Eqs. 28 and 29 that

$$
\begin{aligned}
\lim _{n \rightarrow \infty} \mathbb{P}\left[\forall j: u_{n}\left(M_{n}\left(t_{j}\right)-u_{n}\right) \leq y_{j}\right] \\
\quad=\exp \left(-\int_{\mathbb{R}} \mathbb{P}\left[\exists j: y+W^{(s)}\left(t_{j}\right)-\frac{1}{2} \Gamma\left(t_{j}, s\right)>y_{j}\right] e^{-y} d y\right) .
\end{aligned}
$$

Comparing this with Eq. 19, we obtain the statement of the theorem under the restriction that $\Gamma\left(t_{1}, t_{2}\right)<\infty$ for all $t_{1}, t_{2} \in T$. Note that by Remark 5, we can take $W=W^{(s)}$ on the right-hand side of Eq. 19.

To prove the general case, let $\Gamma$ be a negative-definite kernel in the extended sense, with the corresponding decomposition $T=\cup_{\alpha \in \mathcal{A}} T_{\alpha}$. Take $t_{1}, \ldots, t_{k} \in T$ and $y_{1}, \ldots, y_{k} \in \mathbb{R}$. Let $I_{\alpha}$ be the set of all $i=1, \ldots, k$ with the property that $t_{i} \in T_{\alpha}$. By the Bonferroni inequality,

$$
S_{n}^{(1)}-S_{n}^{(2)} \leq \mathbb{P}\left[\exists j: X_{n}\left(t_{j}\right)>u_{n}\left(y_{j}\right)\right] \leq S_{n}^{(1)}
$$

where

$$
\begin{aligned}
& S_{n}^{(1)}=\sum_{\alpha \in \mathcal{A}} \mathbb{P}\left[\exists j \in I_{\alpha}: X_{n}\left(t_{j}\right)>u_{n}\left(y_{j}\right)\right], \\
& S_{n}^{(2)}=\sum_{\substack{i^{\prime}, i^{\prime \prime}=1, \ldots, k \\
i^{\prime} \nsim i^{\prime \prime}}} \mathbb{P}\left[X_{n}\left(t_{i^{\prime}}\right)>u_{n}\left(y_{i^{\prime}}\right), X_{n}\left(t_{i^{\prime \prime}}\right)>u_{n}\left(y_{i^{\prime \prime}}\right)\right] .
\end{aligned}
$$

(We write $i^{\prime} \sim i^{\prime \prime}$ if $i^{\prime}$ and $i^{\prime \prime}$ are contained in the same $I_{\alpha}$ ). For every $\alpha \in \mathcal{A}$, choose $s_{\alpha} \in T_{\alpha}$ arbitrarily. On the one hand, the above proof, see Eq. 28, shows that for every $\alpha \in \mathcal{A}$,

$$
\begin{aligned}
& \lim _{n \rightarrow \infty} n \mathbb{P}\left[\exists j \in I_{\alpha}: X_{n}\left(t_{j}\right)>u_{n}\left(y_{j}\right)\right] \\
& =\int_{\mathbb{R}} \mathbb{P}\left[\exists j \in I_{\alpha}: y+W^{\left(s_{\alpha}\right)}\left(t_{j}\right)-\frac{1}{2} \Gamma\left(t_{j}, s_{\alpha}\right)>y_{j}\right] e^{-y} d y .
\end{aligned}
$$

On the other hand, we will show that $\lim _{n \rightarrow \infty} n S_{n}^{(2)}=0$. It suffices to prove that if for some $t_{1}, t_{2} \in T, \Gamma\left(t_{1}, t_{2}\right)=\infty$, then for every $y_{1}, y_{2} \in \mathbb{R}$,

$$
p_{n}:=n \mathbb{P}\left[X_{n}\left(t_{i}\right)>u_{n}+u_{n}^{-1} y_{i}, i=1,2\right] \rightarrow 0, \quad n \rightarrow \infty .
$$

Fix $\lambda>0$. Let $\left\{X_{n}^{(\lambda)}(t), t \in\left\{t_{1}, t_{2}\right\}\right\}$ be a zero-mean, unit-variance Gaussian vector with covariance $\rho_{n}:=1-\lambda / \log n$. Recalling Eq. 22 and taking into account the fact that $\Gamma\left(t_{1}, t_{2}\right)=\infty$, we obtain that $r_{n}\left(t_{1}, t_{2}\right)<\rho_{n}$ for sufficiently large $n$. By Slepian's comparison lemma, see Leadbetter et al. (1983, Corollary 4.2.3),

$$
p_{n} \leq n \mathbb{P}\left[X_{n}^{(\lambda)}\left(t_{1}\right)>u_{n}+u_{n}^{-1} y_{1}, X_{n}^{(\lambda)}\left(t_{2}\right)>u_{n}+u_{n}^{-1} y_{2}\right] .
$$


The limit of the right-hand side of Eq. 35 as $n \rightarrow \infty$ has been computed by Hüsler and Reiss (1989). It follows that

$$
\limsup _{n \rightarrow \infty} p_{n} \leq e^{-y_{1}}+e^{-y_{2}}-\Phi\left(\lambda+\frac{y_{2}-y_{1}}{2 \lambda}\right) e^{-y_{1}}-\Phi\left(\lambda+\frac{y_{1}-y_{2}}{2 \lambda}\right) e^{-y_{2}},
$$

where $\Phi$ is the standard normal distribution function. Since the above is true for every $\lambda>0$, we obtain the statement of Eq. 34 by letting $\lambda \rightarrow \infty$.

It follows from Eq. 31 that

$$
\begin{aligned}
& \lim _{n \rightarrow \infty} \mathbb{P}\left[\forall j: M_{n}\left(t_{j}\right) \leq u_{n}\left(y_{j}\right)\right] \\
& \quad=\exp \left(-\sum_{\alpha \in \mathcal{A}} \int_{\mathbb{R}} \mathbb{P}\left[\exists j \in I_{\alpha}: y+W^{\left(s_{\alpha}\right)}\left(t_{j}\right)-\frac{1}{2} \Gamma\left(t_{j}, s_{\alpha}\right)>y_{j}\right] e^{-y} d y\right) .
\end{aligned}
$$

This completes the proof since it follows from Eq. 19 and Section 3.1 that the righthand side is equal to $\mathbb{P}\left[\forall j: M_{\Gamma}\left(t_{j}\right) \leq y_{j}\right]$.

Remark 10 Theorem 2 provides an alternative way to prove the result of Step 1 in the proof of Theorem 1. Let $\left\{X_{n}(t), t \in T\right\}$ be a zero-mean Gaussian process whose covariance function is given by

$$
r_{n}\left(t_{1}, t_{2}\right)=e^{-\Gamma\left(t_{1}, t_{2}\right) /(4 \log n)}, \quad t_{1}, t_{2} \in T .
$$

Note that $r_{n}$ is indeed a valid covariance function by Schoenberg's theorem; see Berg et al. (1984, p.74). The sequence $X_{n}$ satisfies the assumptions of Theorem 2. Taking some $s \in T$ and following the proof of Theorem 2, we arrive at Eq. 30. Since the left-hand side of Eq. 30 does not depend on $s$, it follows that the right-hand side of Eq. 30 does not depend on $s$ either. This implies the statement of Step 1.

\subsection{Necessary and sufficient conditions for convergence}

The next theorem solves Problem 1. To exclude trivial cases, we adopt the following definition: A process $\{M(t), t \in T\}$ is called nondegenerate if there is no $t \in T$ such that $M(t)$ is a.s. constant.

Theorem 3 For every $n \in \mathbb{N}$, let $X_{1 n}, \ldots, X_{n n}$ be independent copies of a zero-mean Gaussian process $\left\{X_{n}(t), t \in T\right\}$ with

$$
r_{n}\left(t_{1}, t_{2}\right):=\mathbb{E}\left[X_{n}\left(t_{1}\right) X_{n}\left(t_{2}\right)\right], \quad \sigma_{n}^{2}(t):=r_{n}(t, t)>0 .
$$

Define a process $\left\{M_{n}(t), t \in T\right\}$ by $M_{n}(t)=\max _{i=1, \ldots, n} X_{i n}(t)$. Then, there exist sequences $a_{n}>0$ and $b_{n}$ such that the process $a_{n}\left(M_{n}-b_{n}\right)$ converges as $n \rightarrow \infty$ to 
a nondegenerate limit $M$ in the sense of finite-dimensional distributions, if and only if, the following two conditions hold:

1. The following limit exists in $[0, \infty]$ for every $t_{1}, t_{2} \in T$ :

$$
\Gamma\left(t_{1}, t_{2}\right):=4 \lim _{n \rightarrow \infty} \log n \cdot\left(1-\frac{r_{n}\left(t_{1}, t_{2}\right)}{\sigma_{n}\left(t_{1}\right) \sigma_{n}\left(t_{2}\right)}\right) ;
$$

2. The following limit exists in $\mathbb{R}$ for every $t_{1}, t_{2} \in T$ :

$$
\kappa\left(t_{1}, t_{2}\right):=2 \lim _{n \rightarrow \infty} \log n \cdot\left(1-\frac{\sigma_{n}\left(t_{1}\right)}{\sigma_{n}\left(t_{2}\right)}\right) .
$$

Remark 11 If Conditions 1 and 2 are satisfied, then we can choose

$$
a_{n}=u_{n} / \sigma_{n}\left(t_{0}\right), \quad b_{n}=\sigma_{n}\left(t_{0}\right) u_{n},
$$

where $t_{0} \in T$ is fixed and $u_{n}$ is as in Eq. 21. In this case, the limiting process $\{M(t), t \in T\}$ has the same law as $\left\{M_{\Gamma}(t)+\kappa\left(t_{0}, t\right), t \in T\right\}$, where $M_{\Gamma}$ is as in Section 3.1.

Remark 12 If we additionally assume that $\sigma_{n} \equiv 1$, then Condition 2 is trivially fulfilled with $\kappa\left(t_{1}, t_{2}\right)=0$.

We will need the following lemma from Kabluchko et al. (2009); see Lemma 21 there.

Lemma 1 Let the assumptions of Theorem 3 be satisfied and, additionally, $\sigma_{n} \equiv 1$. Take some $t_{1}, t_{2} \in T$. Then, the law of the bivariate random vector $\left(u_{n}\left(M_{n}\left(t_{1}\right)-\right.\right.$ $\left.\left.u_{n}\right), u_{n}\left(M_{n}\left(t_{2}\right)-u_{n}\right)\right)$ converges weakly as $n \rightarrow \infty$ if and only if the following limit exists in $[0, \infty]$ :

$$
c:=\lim _{n \rightarrow \infty} \log n \cdot\left(1-r_{n}\left(t_{1}, t_{2}\right)\right)
$$

Proof of Theorem 3 Suppose that the process $a_{n}\left(M_{n}-b_{n}\right)$ converges as $n \rightarrow \infty$ to some nondegenerate limit $M$. First we prove the existence of the limit in Eq. 38. The random variable $\sigma_{n}^{-1}(t) M_{n}(t)$ has the distribution of the maximum of $n$ independent standard Gaussian random variables. Thus, the random variable $u_{n} \sigma_{n}^{-1}(t)\left(M_{n}(t)-\right.$ $\left.\sigma_{n}(t) u_{n}\right)$ converges as $n \rightarrow \infty$ weakly to the Gumbel distribution; see Leadbetter et al. (1983, Theorem 1.5.3). On the other hand, since we assume the process $M$ to be nondegenerate, for every $t \in T$, the random variable $a_{n}\left(M_{n}(t)-b_{n}\right)$ has a nondegenerate limiting distribution as $n \rightarrow \infty$. By the convergence to types lemma 
(see, e.g., Theorem 1.2.3 in Leadbetter et al. 1983), there exist constants $a(t)>0$ and $b(t) \in \mathbb{R}$ such that

$$
\lim _{n \rightarrow \infty} \frac{u_{n} \sigma_{n}^{-1}(t)}{a_{n}}=a(t), \quad \lim _{n \rightarrow \infty} a_{n}\left(\sigma_{n}(t) u_{n}-b_{n}\right)=b(t) .
$$

Inserting $t=t_{1}$ and $t=t_{2}$ into the second equation in Eq. 41 and taking the difference, we obtain

$$
\lim _{n \rightarrow \infty} a_{n} u_{n}\left(\sigma_{n}\left(t_{1}\right)-\sigma_{n}\left(t_{2}\right)\right)=b\left(t_{1}\right)-b\left(t_{2}\right) .
$$

Thus,

$$
\lim _{n \rightarrow \infty} u_{n} a_{n} \sigma_{n}\left(t_{1}\right)\left(1-\frac{\sigma_{n}\left(t_{2}\right)}{\sigma_{n}\left(t_{1}\right)}\right)=b\left(t_{1}\right)-b\left(t_{2}\right) .
$$

Recall that by Eq. 41, $a_{n} \sigma_{n}\left(t_{1}\right) \sim u_{n} / a\left(t_{1}\right)$, and by Eq. $21, u_{n}^{2} \sim 2 \log n$ as $n \rightarrow \infty$. Applying this to Eq. 42, yields Condition 2.

We prove that Condition 1 is satisfied. Take some $t_{1}, t_{2} \in T$ and assume that $t_{1} \neq$ $t_{2}$, since otherwise, the limit in Eq. 37 is evidently 0. Define a unit-variance Gaussian process $\left\{X_{n}^{\prime}(t), t \in T\right\}$ by $X_{n}^{\prime}(t)=X_{n}(t) / \sigma_{n}(t)$. Let $M_{n}^{\prime}(t)=M_{n}(t) / \sigma_{n}(t)$. We claim that the process $u_{n}\left(M_{n}^{\prime}-u_{n}\right)$ converges as $n \rightarrow \infty$ to a nondegenerate limit. Recall that we assume that the process $a_{n}\left(M_{n}-b_{n}\right)$ converges to $M$. We may write

$$
\left\{u_{n}\left(M_{n}^{\prime}(t)-u_{n}\right), t \in T\right\} \stackrel{d}{=}\left\{u_{n} \sigma_{n}^{-1}(t)\left(M_{n}(t)-\sigma_{n}(t) u_{n}\right), t \in T\right\} .
$$

It follows from Eq. 41 that the process on the right-hand side of Eq. 43 converges to $\{a(t)(M(t)-b(t)), t \in T\}$. The covariance function of $X_{n}^{\prime}$ is given by

$$
r_{n}^{\prime}\left(t_{1}, t_{2}\right)=r_{n}\left(t_{1}, t_{2}\right) / \sigma_{n}\left(t_{1}\right) \sigma_{n}\left(t_{2}\right)
$$

Condition 1 follows then from Lemma 1.

The above proves the "only if" part of the theorem. Let us proceed to the proof of the "if" part. Suppose that Conditions 1 and 2 hold. In view of Eq. 44, we may rewrite Eq. 37 as

$$
\Gamma\left(t_{1}, t_{2}\right)=4 \lim _{n \rightarrow \infty} \log n \cdot\left(1-r_{n}^{\prime}\left(t_{1}, t_{2}\right)\right) .
$$

By Theorem 2, the process $u_{n}\left(M_{n}^{\prime}-u_{n}\right)$ converges to $M_{\Gamma}$ as $n \rightarrow \infty$. In particular, the kernel $\Gamma$ is negative definite in the extended sense. With $a_{n}$ and $b_{n}$ as in Eq. 39 we have

$$
\left\{a_{n}\left(M_{n}(t)-b_{n}\right), t \in T\right\} \stackrel{d}{=}\left\{\frac{\sigma_{n}(t)}{\sigma_{n}\left(t_{0}\right)} u_{n}\left(M_{n}^{\prime}(t)-u_{n}\right)-u_{n}^{2}\left(1-\frac{\sigma_{n}(t)}{\sigma_{n}\left(t_{0}\right)}\right), t \in T\right\} .
$$


Note that by Eq. 38,

$$
\lim _{n \rightarrow \infty} \frac{\sigma_{n}(t)}{\sigma_{n}\left(t_{0}\right)}=1, \quad \lim _{n \rightarrow \infty} u_{n}^{2}\left(1-\frac{\sigma_{n}(t)}{\sigma_{n}\left(t_{0}\right)}\right)=-\kappa\left(t_{0}, t\right) .
$$

It follows that the process $a_{n}\left(M_{n}-b_{n}\right)$ converges as $n \rightarrow \infty$ to the process $\left\{M_{\Gamma}(t)+\right.$ $\left.\kappa\left(t_{0}, t\right), t \in T\right\}$. This completes the proof of Remark 11 and the "if" part of the theorem.

\section{Minima of independent Gaussian processes}

In this section we solve Problem 2 using a method similar to that used in Section 3.

\subsection{Construction of limiting processes}

We start by defining the class of limiting processes. Let $\Gamma$ be a negative-definite kernel on a set $T$. Let $\left\{U_{i}, i \in \mathbb{N}\right\}$ be a Poisson point process on $\mathbb{R}$ with Lebesgue measure as intensity, and let $W_{i}, i \in \mathbb{N}$, be independent copies of a zero-mean Gaussian process $\{W(t), t \in T\}$ with incremental variance $\Gamma$ and variance $\sigma^{2}$. Then, we define a process $\left\{L_{\Gamma}(t), t \in T\right\}$ by

$$
L_{\Gamma}(t)=\min _{i \in \mathbb{N}}\left|U_{i}+W_{i}(t)\right|
$$

Remark 13 By Theorem 1 with $\lambda=0$, the law of $L_{\Gamma}$ depends only on $\Gamma$.

Remark 14 It follows from the basic properties of Poisson point processes that the finite-dimensional distributions of $L_{\Gamma}$ are as follows: for every $t_{1}, \ldots, t_{k} \in T$ and $y_{1}, \ldots, y_{k}>0$, we have

$$
\mathbb{P}\left[L_{\Gamma}\left(t_{1}\right)>y_{1}, \ldots, L_{\Gamma}\left(t_{k}\right)>y_{k}\right]=\exp \left(-\int_{\mathbb{R}} \mathbb{P}\left[\exists j:\left|u+W\left(t_{j}\right)\right| \leq y_{j}\right] d u\right) .
$$

For every fixed $t \in T, L_{\Gamma}(t)$ has an exponential distribution with mean $1 / 2$.

Remark 15 The process $L_{\Gamma}$ is max-infinitely divisible, which means that for every $k \in \mathbb{N}$, we can represent $L_{\Gamma}$ as a pointwise minimum of $k$ independent identically distributed processes; see Chapter 5 of Resnick (1987) for a definition. This follows from the fact that the Poisson point process on $\mathbb{R}$ with unit intensity can be represented as a union of $k$ independent Poisson point processes with constant intensity $1 / k$. Note also that the property of max-infinite divisibility is weaker than that of max-stability; cf. Remark 7.

Exactly as in Section 3.1, the above definition of the process $L_{\Gamma}$ can be extended to the case when $\Gamma$ is a negative-definite kernel in the extended sense. 
4.2 Sufficient condition for convergence

The next theorem is an analogue of Theorem 2 for minima (in the sense of absolute value) of independent Gaussian processes. Let

$$
w_{n}=(2 \pi)^{-1 / 2} n .
$$

Theorem 4 For every $n \in \mathbb{N}$, let $X_{1 n}, \ldots, X_{n n}$ be independent copies of a zeromean, unit-variance Gaussian process $\left\{X_{n}(t), t \in T\right\}$ with covariance function $r_{n}\left(t_{1}, t_{2}\right):=\mathbb{E}\left[X_{n}\left(t_{1}\right) X_{n}\left(t_{2}\right)\right]$ such that for every $t_{1}, t_{2} \in T$,

$$
\Gamma\left(t_{1}, t_{2}\right):=\frac{1}{\pi} \lim _{n \rightarrow \infty} n^{2}\left(1-r_{n}\left(t_{1}, t_{2}\right)\right) \in[0, \infty] .
$$

Let $L_{n}(t)=\min _{i=1, \ldots, n}\left|X_{i n}(t)\right|$. Then, the process $w_{n} L_{n}$ converges as $n \rightarrow \infty$ to the process $L_{\Gamma}$ in the sense of finite-dimensional distributions.

Remark 16 By Proposition 1, the kernel $\Gamma$ is negative-definite in the extended sense. Hence, the process $L_{\Gamma}$ is well-defined.

Proof of Theorem 4 The idea of the proof is analogous to that used in the proof of Theorem 2. First suppose that $\Gamma\left(t_{1}, t_{2}\right)<\infty$ for every $t_{1}, t_{2} \in T$. Fix some $s \in T$. Conditioned on the event $A_{n}(y):=\left\{X_{n}(s)=w_{n}^{-1} y\right\}$, we have

$$
\lim _{n \rightarrow \infty} \mathbb{E}\left[w_{n} X_{n}(t) \mid A_{n}(y)\right]=\lim _{n \rightarrow \infty} y r_{n}(t, s)=y .
$$

Further, for every $t_{1}, t_{2} \in T$,

$$
\operatorname{Cov}\left[w_{n} X_{n}\left(t_{1}\right), w_{n} X_{n}\left(t_{2}\right) \mid A_{n}(y)\right]=w_{n}^{2}\left(r_{n}\left(t_{1}, t_{2}\right)-r_{n}\left(t_{1}, s\right) r_{n}\left(t_{2}, s\right)\right)
$$

It follows that

$$
\lim _{n \rightarrow \infty} \operatorname{Cov}\left[w_{n} X_{n}\left(t_{1}\right), w_{n} X_{n}\left(t_{2}\right) \mid A_{n}(y)\right]=\frac{1}{2}\left(\Gamma\left(t_{1}, s\right)+\Gamma\left(t_{2}, s\right)-\Gamma\left(t_{1}, t_{2}\right)\right)
$$

It follows from Eqs. 49 and 50 that for every $y \in \mathbb{R}$, we have the following convergence of conditioned stochastic processes:

$$
\left\{w_{n} X_{n}(t) \mid A_{n}(y), t \in T\right\} \underset{n \rightarrow \infty}{\stackrel{f . d .}{\rightarrow}}\left\{y+W^{(s)}(t), t \in T\right\},
$$

where $W^{(s)}$ is as in Remark 1 . 
Take $k \in \mathbb{N}, t_{1}, \ldots, t_{k} \in T$, and $y_{1}, \ldots, y_{k} \geq 0$. The density $f_{n}$ of the random variable $w_{n} X_{n}(s)$ is given by

$$
f_{n}(y)=\frac{1}{\sqrt{2 \pi} w_{n}} e^{-\frac{y^{2}}{2 w_{n}^{2}}}
$$

Conditioning on the event $A_{n}(y)$, we obtain

$$
\mathbb{P}\left[\exists j: w_{n}\left|X_{n}\left(t_{j}\right)\right| \leq y_{j}\right]=\frac{1}{\sqrt{2 \pi} w_{n}} \int_{\mathbb{R}} \mathbb{P}\left[\exists j: w_{n}\left|X_{n}\left(t_{j}\right)\right| \leq y_{j} \mid A_{n}(y)\right] e^{-\frac{y^{2}}{2 w_{n}^{2}}} d y .
$$

It follows from Eqs. 51 and 52 that

$$
\lim _{n \rightarrow \infty} n \mathbb{P}\left[\exists j: w_{n}\left|X_{n}\left(t_{j}\right)\right| \leq y_{j}\right]=\int_{\mathbb{R}} \mathbb{P}\left[\exists j:\left|y+W^{(s)}\left(t_{j}\right)\right| \leq y_{j}\right] d y
$$

Again, we have omitted the standard justification of the use of the dominated convergence theorem. Recalling that $L_{n}$ is the minimum of $n$ independent copies of $\left|X_{n}\right|$, we obtain

$$
\lim _{n \rightarrow \infty} \mathbb{P}\left[\forall j: w_{n} L_{n}\left(t_{j}\right)>y_{j}\right]=\exp \left(-\int_{\mathbb{R}} \mathbb{P}\left[\exists j:\left|y+W^{(s)}\left(t_{j}\right)\right| \leq y_{j}\right] d y\right)
$$

Comparing this with Eq. 46 (we may take $W=W^{(s)}$ there) completes the proof of the theorem under the restriction that $\Gamma\left(t_{1}, t_{2}\right)$ is finite for all $t_{1}, t_{2} \in T$. The general case can be handled exactly as in the proof of Theorem 2 . We need to show that if for some $t_{1}, t_{2} \in T, \Gamma\left(t_{1}, t_{2}\right)=\infty$, then for every $y_{1}, y_{2}>0$,

$$
p_{n}:=n \mathbb{P}\left[w_{n}\left|X_{n}\left(t_{i}\right)\right|<y_{i}, i=1,2\right] \rightarrow 0, \quad n \rightarrow \infty .
$$

The density $g_{n}$ of the bivariate vector $\left(w_{n} X_{n}\left(t_{1}\right), w_{n} X_{n}\left(t_{2}\right)\right)$ is given by

$$
g_{n}\left(z_{1}, z_{2}\right)=\frac{1}{2 \pi w_{n}^{2} \sqrt{1-r_{n}\left(t_{1}, t_{2}\right)^{2}}} \exp \left(-\frac{z_{1}^{2}+z_{2}^{2}-2 r_{n}\left(t_{1}, t_{2}\right) z_{1} z_{2}}{2 w_{n}^{2}\left(1-r_{n}\left(t_{1}, t_{2}\right)^{2}\right)}\right) .
$$

It follows from Eq. 58 together with $\Gamma\left(t_{1}, t_{2}\right)=\infty$ that the term $\exp (\ldots)$ on the right-hand side converges to 1 as $n \rightarrow \infty$. Furthermore, $\lim _{n \rightarrow \infty} n g_{n}\left(z_{1}, z_{2}\right)=0$. Thus,

$$
p_{n}=\int_{-y_{1}}^{y_{1}} \int_{-y_{2}}^{y_{2}} n g_{n}\left(z_{1}, z_{2}\right) d z_{1} d z_{2} \rightarrow 0, \quad n \rightarrow \infty
$$

This completes the proof of Eq. 53. 
4.3 Necessary and sufficient conditions for convergence

The next theorem solves Problem 2.

Theorem 5 For every $n \in \mathbb{N}$, let $X_{1 n}, \ldots, X_{n n}$ be independent copies of a zero-mean Gaussian process $\left\{X_{n}(t), t \in T\right\}$ with

$$
r_{n}\left(t_{1}, t_{2}\right):=\mathbb{E}\left[X_{n}\left(t_{1}\right) X_{n}\left(t_{2}\right)\right], \quad \sigma_{n}^{2}(t):=r_{n}(t, t)>0 .
$$

Define a process $\left\{L_{n}(t), t \in T\right\}$ by $L_{n}(t)=\min _{i=1, \ldots, n}\left|X_{i n}(t)\right|$. Then, there exists a sequence $a_{n}$ such that the process $a_{n} L_{n}$ converges as $n \rightarrow \infty$ to some nondegenerate limit $L$ in the sense of finite-dimensional distributions, if and only if, the following conditions hold:

1. The following limit exists in $[0, \infty]$ for every $t_{1}, t_{2} \in T$ :

$$
\Gamma\left(t_{1}, t_{2}\right):=\frac{1}{\pi} \lim _{n \rightarrow \infty} n^{2}\left(1-\frac{r_{n}\left(t_{1}, t_{2}\right)}{\sigma_{n}\left(t_{1}\right) \sigma_{n}\left(t_{2}\right)}\right)
$$

2. The following limit exists in $(0, \infty)$ for every $t_{1}, t_{2} \in T$ :

$$
\kappa\left(t_{1}, t_{2}\right):=\lim _{n \rightarrow \infty} \frac{\sigma_{n}\left(t_{1}\right)}{\sigma_{n}\left(t_{2}\right)}
$$

Remark 17 If Conditions 1 and 2 are satisfied, then we can choose

$$
a_{n}=(2 \pi)^{-1 / 2} \sigma_{n}^{-1}\left(t_{0}\right) n
$$

for some fixed $t_{0}$. In this case, the limiting process $\{L(t), t \in T\}$ has the same law as $\left\{\kappa\left(t, t_{0}\right) L_{\Gamma}(t), t \in T\right\}$, where $L_{\Gamma}$ is as in Section 4.1 .

Remark 18 Considering a more general normalization $a_{n}\left(L_{n}-b_{n}\right)$ will not lead to any essentially new processes by the convergence to types lemma.

We will need the following two lemmas.

Lemma 2 Let the assumptions of Theorem 4 be satisfied and, additionally, $\sigma_{n} \equiv 1$. Take some $t_{1}, t_{2} \in T$. The law of the bivariate random vector $\left(w_{n} L_{n}\left(t_{1}\right), w_{n} L_{n}\left(t_{2}\right)\right)$ converges weakly as $n \rightarrow \infty$, if and only if, the following limit exists in $[0, \infty]$ :

$$
c:=\lim _{n \rightarrow \infty} n^{2}\left(1-r_{n}\left(t_{1}, t_{2}\right)\right) .
$$

Proof The "if" part of the lemma follows from Theorem 4. To prove the "only if" part, suppose that the limit on the right-hand side of Eq. 58 does not exist, which means that the sequence $n^{2}\left(1-r_{n}\left(t_{1}, t_{2}\right)\right)$ has at least two different accumulation points $c_{1}, c_{2}$ in $[0, \infty]$. It follows from Theorem 4 that the sequence of vectors 
$\left(w_{n} L_{n}\left(t_{1}\right), w_{n} L_{n}\left(t_{2}\right)\right)$ has at least two weak accumulation points, and these points are different, as it can be seen from the explicit formula Eq. 46. This contradiction completes the proof.

Lemma 3 If $Z_{i}, i \in \mathbb{N}$, are independent standard normal variables, then the random variable $w_{n} \min _{i=1, \ldots, n}\left|Z_{i}\right|$, where $w_{n}$ is as in Eq. 47 , converges as $n \rightarrow \infty$ to the exponential distribution with mean $1 / 2$.

Proof Let $y>0$. Since the density of $Z_{1}$ at 0 is equal to $1 / \sqrt{2 \pi}$, we have

$$
\lim _{n \rightarrow \infty} n \mathbb{P}\left[\left|Z_{1}\right| \leq w_{n}^{-1} y\right]=\lim _{n \rightarrow \infty} \frac{2 w_{n}^{-1} n y}{\sqrt{2 \pi}}=2 y .
$$

It follows that

$$
\lim _{n \rightarrow \infty} \mathbb{P}\left[w_{n} \min _{i=1, \ldots, n}\left|Z_{i}\right|>y\right]=\lim _{n \rightarrow \infty}\left(1-\mathbb{P}\left[\left|Z_{1}\right| \leq w_{n}^{-1} y\right]\right)^{n}=e^{-2 y}
$$

This completes the proof.

Proof of Theorem 5 We prove the "only if" statement of the theorem. Suppose that the process $a_{n} L_{n}$ converges as $n \rightarrow \infty$ to some nondegenerate limit $L$. First we show that Condition 2 holds. The random variable $\sigma_{n}^{-1}(t) L_{n}(t)$ has the distribution of the minimum (in the sense of absolute value) of $n$ independent standard Gaussian random variables. By Lemma 3 , the random variable $w_{n} \sigma_{n}^{-1}(t) L_{n}(t)$ converges as $n \rightarrow \infty$ weakly to the exponential distribution with mean $1 / 2$. On the other hand, by the assumption, the random variable $a_{n} L_{n}(t)$ has a nondegenerate limiting distribution as $n \rightarrow \infty$. By the convergence to types lemma, see Leadbetter et al. (1983, Theorem 1.2.3), there exists a constant $a(t)>0$ such that

$$
\lim _{n \rightarrow \infty} \frac{w_{n} \sigma_{n}^{-1}(t)}{a_{n}}=a(t) .
$$

Using this for $t=t_{1}$ and $t=t_{2}$ and taking the quotient, we obtain Eq. 56.

We prove that Condition 1 holds. Take $t_{1}, t_{2} \in T$ with $t_{1} \neq t_{2}$ (otherwise, the limit in Eq. 55 is 0). Define a unit-variance Gaussian process $\left\{X_{n}^{\prime}(t), t \in T\right\}$ by $X_{n}^{\prime}(t)=X_{n}(t) / \sigma_{n}(t)$, and let $L_{n}^{\prime}(t)=L_{n}(t) / \sigma_{n}(t)$. We show that the process $w_{n} L_{n}^{\prime}$ converges as $n \rightarrow \infty$. We have

$$
\left\{w_{n} L_{n}^{\prime}(t), t \in T\right\} \stackrel{d}{=}\left\{\frac{w_{n} \sigma_{n}^{-1}(t)}{a_{n}} a_{n} L_{n}(t), t \in T\right\} .
$$

It follows from Eq. 59 that the process $w_{n} L_{n}^{\prime}$ converges as $n \rightarrow \infty$ to $\{a(t) L(t), t \in$ $T$ \}. Condition 1 follows then from Lemma 2. 
To prove the "if" part of the theorem, note that if Condition 1 is satisfied, then $r_{n}^{\prime}$, the covariance function of $X_{n}^{\prime}$, satisfies

$$
\frac{1}{\pi} \lim _{n \rightarrow \infty} n^{2} \cdot\left(1-r_{n}^{\prime}\left(t_{1}, t_{2}\right)\right)=\Gamma\left(t_{1}, t_{2}\right)
$$

By Theorem 4, the process $w_{n} L_{n}^{\prime}$ converges to $L_{\Gamma}$ as $n \rightarrow \infty$. If $a_{n}$ is chosen as in Eq. 57, then

$$
\left\{a_{n} L_{n}(t), t \in T\right\} \stackrel{d}{=}\left\{\frac{\sigma_{n}(t)}{\sigma_{n}\left(t_{0}\right)} w_{n} L_{n}^{\prime}(t), t \in T\right\} .
$$

It follows from Condition 2 that the process on the right-hand side converges as $n \rightarrow \infty$ to the process $\left\{\kappa\left(t, t_{0}\right) L_{\Gamma}(t), t \in T\right\}$. This completes the proof.

\section{Sufficient conditions for tightness}

Theorem 3 provides necessary and sufficient conditions for the weak convergence of the finite-dimensional distributions of the pointwise maximum $M_{n}$ of independent Gaussian processes. In this section we provide a sufficient condition for tightness of the appropriately normalized sequence $M_{n}$ on $C(T)$, the space of continuous functions on $T$. Combining both results establishes the weak convergence on $C(T)$; see Chapter 2 of Billingsley (1999). A criterion for the weak convergence of pointwise maxima of i.i.d. sequences of stochastic processes has been established in de Haan and Lin (2001). Note that we are considering triangular arrays (which is a more general setting than the i.i.d. sequences). The reason is that for Gaussian processes, i.i.d. sequences do not lead to non-trivial limits.

We assume that the processes under consideration are defined on a compact metric space $(T, d)$ and have a.s. continuous paths. Let $N(\varepsilon)$ be smallest number of balls of radius $\varepsilon>0$ needed to cover the space $T$. We will need the following entropy condition:

$$
\int_{0}^{1}(\log N(\varepsilon))^{1 / 2} d \varepsilon<\infty
$$

Theorem 6 For every $n \in \mathbb{N}$, let $X_{1 n}, \ldots, X_{n n}$ be independent, a.s. continuous copies of a zero-mean Gaussian process $\left\{X_{n}(t), t \in T\right\}$ defined on a compact metric space $(T, d)$ satisfying Eq. 60. Let $r_{n}\left(t_{1}, t_{2}\right):=\mathbb{E}\left[X_{n}\left(t_{1}\right) X_{n}\left(t_{2}\right)\right]$ and $\sigma_{n}^{2}(t):=$ $r_{n}(t, t)>0$. Assume that there is $C>0$ such that for every $t_{1}, t_{2} \in T$,

$$
\begin{array}{r}
\log n \cdot\left(1-\frac{r_{n}\left(t_{1}, t_{2}\right)}{\sigma_{n}\left(t_{1}\right) \sigma_{n}\left(t_{2}\right)}\right)<C d\left(t_{1}, t_{2}\right), \\
\log n \cdot\left(1-\frac{\sigma_{n}\left(t_{1}\right)}{\sigma_{n}\left(t_{2}\right)}\right)<C d\left(t_{1}, t_{2}\right) .
\end{array}
$$


Let $M_{n}(t)=\max _{i=1, \ldots, n} X_{i n}(t)$. Define $u_{n}$ as in Eq. 21. Then, for arbitrary $t_{0} \in T$, the sequence of processes $\sigma_{n}\left(t_{0}\right)^{-1} u_{n}\left(M_{n}-\sigma_{n}\left(t_{0}\right) u_{n}\right)$ is tight on $C(T)$.

Proof Let us start by showing that we may assume without loss of generality that $\sigma_{n}(t)=1$ for all $t \in T$. Fix some $t_{0} \in T$. Define the standardized processes $X_{n}^{\prime}(t)=$ $X_{n}(t) / \sigma_{n}(t)$ and $M_{n}^{\prime}(t)=M_{n}(t) / \sigma_{n}(t)$. We have

$$
\sigma_{n}\left(t_{0}\right)^{-1} u_{n}\left(M_{n}(t)-\sigma_{n}\left(t_{0}\right) u_{n}\right)=\frac{\sigma_{n}(t)}{\sigma_{n}\left(t_{0}\right)} u_{n}\left(M_{n}^{\prime}(t)-u_{n}\right)-\kappa_{n}(t),
$$

where $\kappa_{n}(t)=u_{n}^{2}\left(1-\frac{\sigma_{n}(t)}{\sigma_{n}\left(t_{0}\right)}\right)$. Recall that by Eq. $21, u_{n}^{2} \sim 2 \log n$ as $n \rightarrow \infty$. It follows from Eq. 62 that

$$
\lim _{n \rightarrow \infty} \frac{\sigma_{n}(t)}{\sigma_{n}\left(t_{0}\right)}=1 \text { uniformly in } t \in T
$$

We are going to show that the sequence of functions $\kappa_{n}, n \in \mathbb{N}$, is relatively compact in $C(T)$. By Eq. 62, the sequence $\kappa_{n}$ is uniformly bounded. By the Arzelà-Ascoli theorem, we need to show that the family $\kappa_{n}, n \in \mathbb{N}$, is equicontinuous. It follows from Eq. 64 that there is $c_{1}$ such that $\sigma_{n}(t)<c_{1} \sigma_{n}\left(t_{0}\right)$ for all $n \in \mathbb{N}, t \in T$. Again by Eq. 62 ,

$$
u_{n}^{2}\left(\sigma_{n}\left(t_{2}\right)-\sigma_{n}\left(t_{1}\right)\right) \leq 3 C d\left(t_{1}, t_{2}\right) \sigma_{n}\left(t_{2}\right) \leq 3 c_{1} C d\left(t_{1}, t_{2}\right) \sigma_{n}\left(t_{0}\right), \quad n>N
$$

Recalling the definition of $\kappa_{n}$, we obtain that $\kappa_{n}\left(t_{1}\right)-\kappa_{n}\left(t_{2}\right) \leq c_{2} d\left(t_{1}, t_{2}\right)$ for some constant $c_{2}$. This proves that the family of functions $\kappa_{n}, n \in \mathbb{N}$, is equicontinuous.

From Eq. 63, Eq. 64 and the relative compactness of the family $\kappa_{n}, n \in \mathbb{N}$, it follows that to prove the theorem, it suffices to show that the sequence $u_{n}\left(M_{n}^{\prime}(t)-u_{n}\right)$ is tight.

For the rest of the proof, we assume that $\sigma_{n}(t)=1$ for all $t \in T$ and replace condition Eq. 61 by the following one:

$$
\log n \cdot\left(1-r_{n}\left(t_{1}, t_{2}\right)\right)<C d\left(t_{1}, t_{2}\right)
$$

Recall that $t_{0} \in T$ is fixed. For $y \in \mathbb{R}$, denote by $X_{n}^{(y)}$ the process $u_{n}\left(X_{n}-u_{n}\right)-y$ conditioned on the event $A_{n}(y)=\left\{X_{n}\left(t_{0}\right)=u_{n}+u_{n}^{-1} y\right\}$. A careful inspection shows that the tightness part of the proof of Theorem 17 in Kabluchko et al. (2009) applies in the more general setting of Theorem 6, provided that the following conditions have been verified:

1. The family of processes $X_{n}^{(y)}-\mathbb{E}\left[X_{n}^{(y)}\right], y \in \mathbb{R}, n \in \mathbb{N}$, is tight on $C(T)$;

2. For every $c>0$, the family of processes $X_{n}^{(y)}, y \in[-c, c], n \in \mathbb{N}$, is tight on $C(T)$. 
We concentrate on verifying these conditions. Given a function $f \in C(T)$, define its continuity modulus by

$$
\omega_{\delta}(f)=\sup _{\substack{t_{1}, t_{2} \in T \\ d\left(t_{1}, t_{2}\right) \leq \delta}}\left|f\left(t_{1}\right)-f\left(t_{2}\right)\right|, \quad \delta>0 .
$$

Note that $X_{n}^{(y)}\left(t_{0}\right)=0$. We will use a standard tightness criterion; see Theorem 7.3 in Billingsley (1999). To prove the first condition, it suffices to show that for every $a>0$, there is $\delta=\delta(a)$ such that

$$
\mathbb{P}\left[\omega_{\delta}\left(X_{n}^{(y)}-\mathbb{E}\left[X_{n}^{(y)}\right]\right)>a\right]<a, \quad \text { for all } n \in \mathbb{N}, y \in \mathbb{R}
$$

By the formula for the conditional variance of Gaussian processes, we have

$$
\begin{aligned}
\operatorname{Var}\left(X_{n}^{(y)}\left(t_{1}\right)-X_{n}^{(y)}\left(t_{2}\right)\right) & =u_{n}^{2}\left(2-2 r_{n}\left(t_{1}, t_{2}\right)-\left(r_{n}\left(t_{1}, t_{0}\right)-r_{n}\left(t_{2}, t_{0}\right)\right)^{2}\right) \\
& \leq 2 u_{n}^{2}\left(1-r_{n}\left(t_{1}, t_{2}\right)\right) .
\end{aligned}
$$

Together with Eq. 65 this implies that

$$
\operatorname{Var}\left(X_{n}^{(y)}\left(t_{1}\right)-X_{n}^{(y)}\left(t_{2}\right)\right) \leq 5 C d\left(t_{1}, t_{2}\right)
$$

Note that the right-hand side does not depend on $n \in \mathbb{N}, y \in \mathbb{R}$. By an estimate for the continuity modulus of a zero-mean Gaussian process in terms of its canonical distance (see, e.g., Corollary 1.3.4 of Adler and Taylor 2007), it follows that for some constant $K$,

$$
\mathbb{E}\left[\omega_{\delta}\left(X_{n}^{(y)}-\mathbb{E}\left[X_{n}^{(y)}\right]\right)\right] \leq K \int_{0}^{\delta}\left(\log N\left((5 C)^{-1} \varepsilon\right)\right)^{1 / 2} d \varepsilon, \quad \delta \in(0,1) .
$$

Since the integral on the right-hand side is convergent by Eq. 60, we may choose $\delta$ so small that $\mathbb{E}\left[\omega_{\delta}\left(X_{n}^{(y)}-\mathbb{E}\left[X_{n}^{(y)}\right]\right)\right]<a^{2}$. Applying Markov's inequality, we obtain Eq. 66. This completes the verification of the first condition.

To prove the second condition, it suffices to verify that the family of functions $\mu_{n}^{(y)}, y \in[-c, c], n \in \mathbb{N}$, where $\mu_{n}^{(y)}(t)=-\mathbb{E}\left[X_{n}^{(y)}(t)\right]$, is relatively compact in $C(T)$. By the formula for the conditional expectation of Gaussian processes, we have

$$
\mu_{n}^{(y)}(t)=-\mathbb{E}\left[X_{n}^{(y)}(t)\right]=\left(u_{n}^{2}+y\right)\left(1-r_{n}\left(t, t_{0}\right)\right)
$$

It follows from Eqs. 65 and 67 that the family $\mu_{n}^{(y)}, y \in[-c, c], n \in \mathbb{N}$, is uniformly bounded. Let us show that the family of functions $\left(\mu_{n}^{(y)}\right)^{1 / 2}, y \in[-c, c], n \in \mathbb{N}$, 
is equicontinuous. The pseudometric $d_{n}$ generated by the Gaussian process $X_{n}$ is defined by

$$
d_{n}^{2}\left(t_{1}, t_{2}\right)=\operatorname{Var}\left(X_{n}\left(t_{1}\right)-X_{n}\left(t_{2}\right)\right)=2\left(1-r_{n}\left(t_{1}, t_{2}\right)\right)
$$

It follows from Eq. 67, the triangle inequality, and Eq. 65 that

$$
\begin{aligned}
\left(\mu_{n}^{(y)}\left(t_{1}\right)\right)^{1 / 2}-\left(\mu_{n}^{(y)}\left(t_{2}\right)\right)^{1 / 2} & =2^{-1 / 2}\left(u_{n}^{2}+y\right)^{1 / 2}\left(d_{n}\left(t_{0}, t_{1}\right)-d_{n}\left(t_{0}, t_{2}\right)\right) \\
& \leq 2^{-1 / 2}\left(u_{n}^{2}+y\right)^{1 / 2} d_{n}\left(t_{1}, t_{2}\right) \\
& =\left(u_{n}^{2}+y\right)^{1 / 2}\left(1-r_{n}\left(t_{1}, t_{2}\right)\right)^{1 / 2} \\
& \leq 3 C d\left(t_{1}, t_{2}\right),
\end{aligned}
$$

where in the last inequality we have used that $u_{n}^{2}+y \leq 3 \log n$ as long as $y \in$ $[-c, c]$ and $n$ is large. The family of functions $\left(\mu_{n}^{(y)}(t)\right)^{1 / 2}, y \in[-c, c], n \in \mathbb{N}$, is equicontinuous and also uniformly bounded. Consequently, the family $\mu_{n}^{(y)}(t)$, $y \in[-c, c], n \in \mathbb{N}$, is relatively compact in $C(T)$. This completes the verification of the second condition.

\section{Examples}

6.1 Maxima of independent fractional Brownian motions

Recall that a zero-mean Gaussian process $\{B(t), t \in \mathbb{R}\}$ is called a fractional Brownian motion with index $\alpha \in(0,2)$ if

$$
\operatorname{Cov}\left(B\left(t_{1}\right), B\left(t_{2}\right)\right)=\frac{1}{2}\left(\left|t_{1}\right|^{\alpha}+\left|t_{2}\right|^{\alpha}-\left|t_{1}-t_{2}\right|^{\alpha}\right) .
$$

The next theorem describes the limiting processes for maxima of independent fractional Brownian motions. For the standard Brownian motion (which corresponds to $\alpha=1$ ), it reduces to a result of Brown and Resnick (1977) (whose proof was based on the Markov property of the Brownian motion). On the other hand, maxima of independent stationary Gaussian processes have been studied by Kabluchko et al. (2009).

Theorem 7 Let $B_{i}, i \in \mathbb{N}$, be independent copies of a fractional Brownian motion $\{B(t), t \in \mathbb{R}\}$ with index $\alpha \in(0,2)$. Define $u_{n}$ as in Eq. 21. Let $s_{n}=(2 \log n)^{-1 / \alpha}$ if $\alpha \in(0,1]$ and $s_{n}=(2 \log n)^{-1}$ if $\alpha \in[1,2)$. Define

$$
M_{n}(t)=\max _{i=1, \ldots, n} B_{i}\left(1+s_{n} t\right)
$$


For every compact set $K \subset \mathbb{R}$, the following convergence of stochastic processes holds weakly on $C(K)$ :

$$
\left\{u_{n}\left(M_{n}(t)-u_{n}\right), t \in K\right\} \underset{n \rightarrow \infty}{\stackrel{w}{\longrightarrow}} \begin{cases}\left\{M_{\Gamma}(t), t \in K\right\}, & \text { if } \alpha \in(0,1), \\ \left\{M_{\Gamma}(t)+t / 2, t \in K\right\}, & \text { if } \alpha=1, \\ \{G+\alpha t / 2, t \in K\}, & \text { if } \alpha \in(1,2) .\end{cases}
$$

Here, $M_{\Gamma}$ is defined as in Section 3.1 with $\Gamma\left(t_{1}, t_{2}\right)=\left|t_{1}-t_{2}\right|^{\alpha}$, and $G$ is a random variable with Gumbel distribution, that is $\mathbb{P}[G \leq y]=e^{-e^{-y}}$ for every $y \in \mathbb{R}$.

Remark 19 An additional rescaling represented by the sequence $s_{n}$ is needed to obtain a nontrivial limit; cf. Brown and Resnick (1977), Kabluchko et al. (2009). It can be shown that the above choice of $s_{n}$ is (up to a multiplicative constant) unique: any other choice does not lead to any interesting limit.

Proof of Theorem 7 To prove the weak convergence of the finite-dimensional distributions in Eq. 70, we are going to apply Theorem 3 with $X_{n}(t)=B\left(1+s_{n} t\right)$. The tightness in $C(K)$ will be established using Theorem 6 . Note that $\lim _{n \rightarrow \infty} s_{n}=0$. By Eq. 68, the variance $\sigma_{n}^{2}(t):=\operatorname{Var} X_{n}(t)$ satisfies for every $t_{1}, t_{2} \in \mathbb{R}$,

$$
1-\frac{\sigma_{n}\left(t_{1}\right)}{\sigma_{n}\left(t_{2}\right)}=1-\left(\frac{1+s_{n} t_{1}}{1+s_{n} t_{2}}\right)^{\alpha / 2}=\frac{\alpha}{2}\left(t_{2}-t_{1}\right) s_{n}(1+o(1)), \quad n \rightarrow \infty .
$$

In a similar way, we obtain that $r_{n}\left(t_{1}, t_{2}\right):=\mathbb{E}\left[X_{n}\left(t_{1}\right) X_{n}\left(t_{2}\right)\right]$, the covariance function of $X_{n}$, satisfies

$$
\begin{aligned}
1-\frac{r_{n}\left(t_{1}, t_{2}\right)}{\sigma_{n}\left(t_{1}\right) \sigma_{n}\left(t_{2}\right)} & =1-\frac{1}{2}\left(\frac{\sigma_{n}\left(t_{1}\right)}{\sigma_{n}\left(t_{2}\right)}+\frac{\sigma_{n}\left(t_{2}\right)}{\sigma_{n}\left(t_{1}\right)}-\frac{\left|t_{1}-t_{2}\right| s_{n}^{\alpha}}{\sigma_{n}\left(t_{1}\right) \sigma_{n}\left(t_{2}\right)}\right) \\
& =\frac{1}{2}\left|t_{1}-t_{2}\right|^{\alpha} s_{n}^{\alpha}(1+o(1)), \quad n \rightarrow \infty .
\end{aligned}
$$

Let us prove the weak convergence of the finite-dimensional distributions in Eq. 70. It follows from Eqs. 71 and 72 that Conditions 1 and 2 of Theorem 3 are satisfied with

$$
\Gamma\left(t_{1}, t_{2}\right)=\left\{\begin{array}{ll}
\left|t_{1}-t_{2}\right|^{\alpha}, & \text { if } \alpha \in(0,1], \\
0, & \text { if } \alpha \in(1,2),
\end{array} \quad \kappa\left(t_{1}, t_{2}\right)= \begin{cases}0, & \text { if } \alpha \in(0,1), \\
\alpha\left(t_{2}-t_{1}\right) / 2, & \text { if } \alpha \in[1,2)\end{cases}\right.
$$

Theorem 3 and Remark 11 imply that Eq. 70 holds in the sense of weak convergence of finite-dimensional distributions. [Note that if $\Gamma \equiv 0$, then $M_{\Gamma} \equiv G$, where $G$ is Gumbel distributed]. 
To prove the weak convergence in $C(K)$, note that Eqs. 71 and 72 hold uniformly in $t_{1}, t_{2} \in K$. This implies that Conditions 61, 62 of Theorem 6 are satisfied with $d\left(t_{1}, t_{2}\right)=\left|t_{1}-t_{2}\right|^{\alpha}$ if $\alpha \in(0,1]$ and $d\left(t_{1}, t_{2}\right)=\left|t_{1}-t_{2}\right|$ if $\alpha \in[1,2)$.

\subsection{Minima of independent fractional Brownian motions}

The next theorem describes possible limits for minima of independent fractional Brownian motions. In contrast with Theorem 7 , there is no change in behavior at $\alpha=1$ here.

Theorem 8 Let $B_{i}, i \in \mathbb{N}$, be independent copies of a fractional Brownian motion $\{B(t), t \in \mathbb{R}\}$ with index $\alpha \in(0,2)$. With $s_{n}=\left(2 \pi / n^{2}\right)^{1 / \alpha}$, define

$$
L_{n}(t)=\min _{i=1, \ldots, n}\left|B_{i}\left(1+s_{n} t\right)\right|
$$

For every compact set $K \subset \mathbb{R}$, the process $(2 \pi)^{-1 / 2} n L_{n}$ converges as $n \rightarrow \infty$ to the process $\left\{L_{\Gamma}(t), t \in \mathbb{R}\right\}$ weakly on $C(K)$. Here, $L_{\Gamma}$ is defined as in Section 4.1 with $\Gamma\left(t_{1}, t_{2}\right)=\left|t_{1}-t_{2}\right|^{\alpha}$.

Proof We will prove only the convergence of the finite-dimensional distributions. We apply Theorem 5 to the process $X_{n}(t)=B\left(1+s_{n} t\right)$. As in the proof of Theorem 7 , the variance and the covariance of $X_{n}$ satisfy Eqs. 71 and 72. It follows that Conditions 1 and 2 of Theorem 5 hold with $\kappa\left(t_{1}, t_{2}\right)=1$ and $\Gamma\left(t_{1}, t_{2}\right)=\left|t_{1}-t_{2}\right|^{\alpha}$.

Remark 20 It is possible to obtain a generalization of Theorem 4, Theorem 5 and Theorem 8 to $\chi^{2}$-processes. A $d$-dimensional $\chi^{2}$-process is defined as the Euclidean norm of an $\mathbb{R}^{d}$-valued process with i.i.d. Gaussian components. This, in particular, recovers the results of Penrose $(1988,1991)$, where minima of independent Bessel processes have been studied. Note that the proofs of Penrose (1988, 1991) are based on the Markov property of Bessel processes. Global minima of stationary $\chi^{2}$-processes have been studied by Albin (1996).

Acknowledgement The author is grateful to Martin Schlather for useful remarks.

\section{References}

Adler, R.J., Taylor, J.E.: Random fields and geometry. In: Springer Monographs in Mathematics. Springer, New York (2007)

Albin, J.M.P.: Minima of $H$-valued Gaussian processes. Ann. Probab. 24(2), 788-824 (1996)

Berg, C., Christensen, J.P.R., Ressel, P.: Harmonic analysis on semigroups. Theory of Positive Definite and Related Functions. In: Graduate Texts in Mathematics, vol. 100. Springer, New York (1984)

Billingsley, P.: Convergence of probability measures, 2nd edn. In: Wiley Series in Probability and Statistics: Probability and Statistics. Wiley, New York (1999)

Brown, B.M., Resnick, S.I.: Extreme values of independent stochastic processes. J. Appl. Probab. 14, $732-739$ (1977)

de Haan, L.: A spectral representation for max-stable processes. Ann. Probab. 12, 1194-1204 (1984) 
de Haan, L., Lin, T.: On convergence toward an extreme value distribution in $C[0,1]$. Ann. Probab. 29(1), 467-483 (2001)

Falk, M., Hüsler, J., Reiss, R.-D.: Laws of small numbers: extremes and rare events. In: DMV Seminar, vol. 23. Birkhäuser, Basel (1994)

Hüsler, J., Reiss, R.-D.: Maxima of normal random vectors: between independence and complete dependence. Stat. Probab. Lett. 7(4), 283-286 (1989)

Istas, J.: Spherical and hyperbolic fractional Brownian motion. Electron. Commun. Probab. 10, 254-262 (2005)

Kabluchko, Z., Schlather, M., de Haan, L.: Stationary max-stable fields associated to negative definite functions. Ann. Probab. 37(5), 2042-2065 (2009)

Leadbetter, M.R., Lindgren, G., Rootzén, H.: Extremes and related properties of random sequences and processes. In: Springer Series in Statistics. Springer, New York (1983)

Penrose, M.: The minimum of a large number of Bessel processes. J. Lond. Math. Soc. II. 38(3), 566-576 (1988)

Penrose, M.: Minima of independent Bessel processes and of distances between Brownian particles. J. Lond. Math. Soc. II. 43(2), 355-366 (1991)

Pickands, J.: Upcrossing probabilities for stationary Gaussian processes. Trans. Am. Math. Soc. 145, 51-73 (1969)

Piterbarg, V.I.: Asymptotic methods in the theory of Gaussian processes and fields. In: Translations of Mathematical Monographs, vol. 148. American Mathematical Society, Providence (1996)

Resnick, S.I.: Extreme values, regular variation, and point processes. In: Applied Probability, vol. 4. Springer, New York (1987) 